\title{
Dog Care Center in Badung, Bali Application of Harmony Concept in Space Design in Dog Custody
}

\author{
Bachelor Degree of Architecture Study Program \\ Faculty of Engineering-Udayana University \\ Jimbaran \\ Aldy.susantoep@gmail.com \\ Architecture Study Program \\ Faculty of Engineering-Udayana University \\ Jimbaran \\ Architecture Study Program \\ Faculty of Engineering-Udayana University \\ Jimbaran
}

I Gede Aldy Susanto Eka Putra ${ }^{1}$, Ni Ketut Agusintadewi ${ }^{2}$, I Gusti Agung Bagus Suryada ${ }^{3}$

\begin{abstract}
Dogs are animals that have high popularity as pets in the global community. Indonesia is no exception, but people still do not understand the responsibility for caring dogs as pets. This has an impact on the dog's assumption only as a comforter without thinking about the importance of their needs and care. Because there is a considerable comparison between the level of dog popularity and the sense of responsibility for caring dogs as pets, a place is needed to harmonize the gap. The Dog Care Center is needed to educate the public and provide facilities for dog needs and care, with the theme of Natural Living design that takes the concept of Harmony in the design of the interior. The choice of harmony concept has an emphasis on the design of space, material and color that can give the impression of openness without the separation between human and dog in it.
\end{abstract}

Index Terms — dog, care, center, harmony.

\section{INTRODUCTION}

The closeness between dogs and humans is not something that has recently developed. The Balinese also have a philosophy that bases the closeness of the Balinese people with dogs. This can be seen in the story of Panca Padawa's journey in exile to a forest accompanied by a white dog. In the story illustrates how faithful a dog is to its masaji and also in Balinese society the dog has a noble role, because according to existing culture and folklore, a dog will deliver humans later into heaven according to the story to the local community. This is what underlies the closeness of the Balinese community with dogs.

Ownership of dogs in Balinese society, apart from a sense of love for dogs is also based on that philosophy. Data according to the Bali Provincial Animal Husbandry Service and guest book recapitulation from dog care services in the Badung Regency area, the total number of dog population in 2016 reached 411,153 more with the percentage of wild dogs $5 \%$ and $95 \%$ ownership dogs with a total of 390,595 tails. The distribution of dog population in Badung Regency alone reached 56,394 tails. Whereas the availability of a dog care center is not proportional to the number of dogs available.

While the availability of dog daycare or dog hotels overall does not reach 100 places and is spread across nine districts. In Badung Regency, there are four belsa dog care centers with an average of only thirty dogs. Judging from the above cases, it is very necessary to develop a dog care facility which has complete facilities according to the existing standards, so it can reduce the dog's neglect due to the owner's activity and the dog owner can carry out his activities smoothly without any burden on the condition of 
his pet.

\section{RESUlt AND ANALISYS}

\section{A. Theme of Design}

The design of the dog care center takes the theme of Natural Living with the aim of designing buildings that do not disturb and damage existing conditions and ecosystems. The choice of theme is based on the rapid development of development but only determines the function of the building without regard to the condition of the surrounding ecosystems such as the lack of green land and infiltration areas which can have a negative impact.

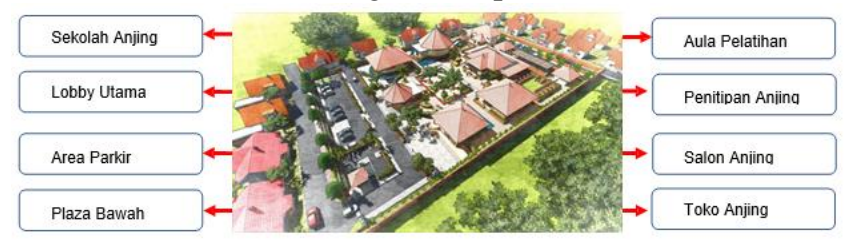

Fig. 1. Theme (source: Putra:2017)

The theme of Natural Living literally means life or activity in harmony with natural conditions without disturbing or damaging existing nature[1]. The emphasis on building design emphasizes the proportion of space with open areas and catchment areas as parks or entertainment zones.

\section{B. Concept}

The concept taken from the Natural Living Theme is the concept of Harmony. The concept of harmony is a design concept that focuses on the harmony of buildings with the surrounding environment. When this concept is associated with a dining building, it becomes a building design that reflects balance and equality. Harmony is more dominant in the use of natural materials and natural soft colors to create nuances that blend with nature.

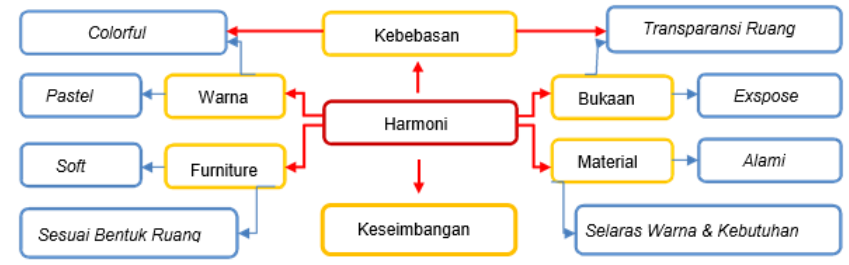

Fig. 2. Concept

\section{Harmony Concept in Designing The Space}

Through the concept of harmony in the design of space in emphasizing balance and freedom so that there is a design rhythm that is not monotonous but does not crash into each other's designs but is interconnected. The design of space in a dog care center is designed with an emphasis on freedom in the sense that it does not provide a large limit between the space for the human and dog civitas while still considering the comfort of both. It aims to make the civitas can play and play freely.

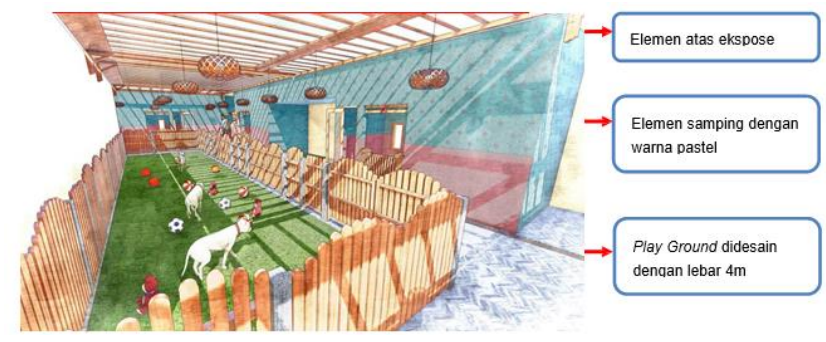

Fig. 3. Interior Concept

The design of space in the dog room care is designed using elements of the exposure aims to maximize the need for light for dog health even though it remains in the room. Laying the elements of this exposure is right above the dog playground so that when playing dogs can also be exposed to sufficient light for their health aspects. Playground is designed with a size of $12 \mathrm{mx} 4 \mathrm{~m}$ for freedom and freedom of play dogs and can participate in humans there.

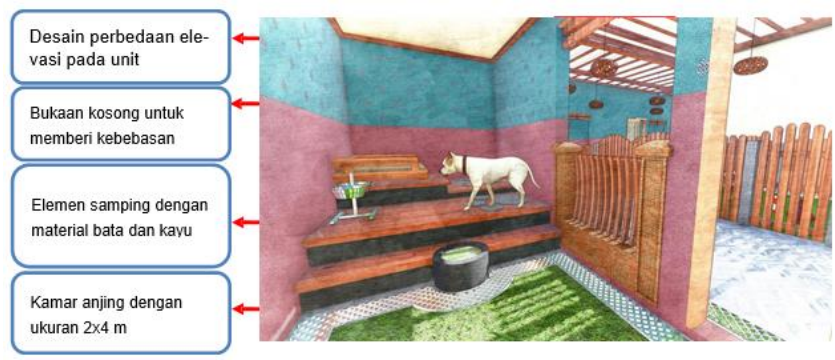

Fig. 4. Interior Concept

In the day care unit designed with a floor elevation game, it aims to provide different functions for each elevation. The dog will choose an elevation that is closer to the ceiling as a place to sleep because it feels more comfortable and safe. In addition, the application of the concept of harmony to the freedom of dogs is applied to a wide open system without glass, thereby reducing the impression of imprisoning a dog inside. Equipped with synthetic grass so it gives a natural impression. The dog care unit is designed with a size of $4 \mathrm{~m}$ $\mathrm{x} 2 \mathrm{~m}$ to have a spacious atmosphere and the dog does not get bored to be in it. The color used is also varied with the pastel color group and the combination of wood and brick material as an addition to its natural feel. It was designed to create an atmosphere like being outdoors even though indoors. Around the grass area is equipped with a recharge system because in general dogs will choose a grassy area to get rid of their waste. The human circulation path is designed with a width of $3 \mathrm{~m}$ to simplify the maintenance process. In overcoming unwanted things all of the side elements are coated with epoxy to anticipate the marking habit in dogs and make it easier in the cleaning process so that the side elements do not become moist.

\section{CONCLUSION}

The dog care center is a multi-functional commercial facility by providing complex services for various needs in dog care and at the same time as educating the importance of understanding in caring for and maintaining the health of their pet dogs which so far have not been understood well 
by the wider community. This dog care center is designed with the theme Natural Living given the rapid development of modern development that has minimal maintenance and concern for the surrounding environment.

The theme and concept of the design was developed in accordance with what was intended, namely planning a place that could unite the human community with pet dogs and at the same time educate the community about the importance of coexistence and mutual understanding of the environment so that the concept of harmony was chosen in the design of this dog care center. The concept of harmony in question is to harmonize the relationship between human dogs and nature as the environment. In the design of this concept design is emphasized on the design of buildings that have an open impression does not imprison the dog and provide proper comfort for humans and dogs in it. The interior design is also made to be able to enjoy outdoor arrangement even though it remains outside the room so that the interior design seems semi open space.

The advice given in the design of this dog care center is to make a decent space without breaking down the civitas and seem open and free both for humans and humans because they both live side by side. The designed space must reduce the nuances of stagnating but to stay in the same activity in one room by considering the comfort of all parties.

\section{REFERENCES}

[1] Barr, Vilma \& C. E. Broudy, "Designing To Sell”, 1984. 